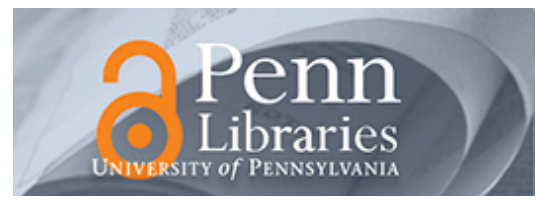

University of Pennsylvania

ScholarlyCommons

$5-1-1996$

\title{
Magnetic Anisotropies and General On-Site Coulomb Interactions in the Cuprates
}

Ora Entin-Wohlman

A. Brooks Harris

University of Pennsylvania, harris@sas.upenn.edu

Amnon Aharony

Follow this and additional works at: https://repository.upenn.edu/physics_papers

Part of the Physics Commons

\section{Recommended Citation}

Entin-Wohlman, O., Harris, A., \& Aharony, A. (1996). Magnetic Anisotropies and General On-Site Coulomb Interactions in the Cuprates. Physical Review B, 53 (17), 11661-11670. http://dx.doi.org/10.1103/

PhysRevB.53.11661

At the time of publication, author A. Brooks Harris was also affiliated with Tel Aviv University, Tel Aviv, Israel. Currently, he is a faculty member in the Physics Department at the University of Pennsylvania.

This paper is posted at ScholarlyCommons. https://repository.upenn.edu/physics_papers/347

For more information, please contact repository@pobox.upenn.edu. 


\title{
Magnetic Anisotropies and General On-Site Coulomb Interactions in the Cuprates
}

\author{
Abstract \\ This paper derives the anisotropic superexchange interactions from a Hubbard model for excitations \\ within the copper $3 d$ band and the oxygen $2 p$ band of the undoped insulating cuprates. We extend the \\ recent calculation of Yildirim et al. [Phys. Rev. B 52, 10239 (1995)] in order to include the most general \\ on-site Coulomb interactions (including those which involve more than two orbitals) when two holes \\ occupy the same site. Our general results apply when the oxygen ions surrounding the copper ions form \\ an octahedron which has tetragonal symmetry (but may be rotated as in lanthanum cuprate). For the \\ tetragonal cuprates we obtain an easy-plane anisotropy in good agreement with experimental values. We \\ predict the magnitude of the small in-plane anisotropy gap in the spin-wave spectrum of $\mathrm{YBa}_{2} \mathrm{Cu}_{3} \mathrm{O}_{6}$. \\ Disciplines \\ Physics \\ Comments \\ At the time of publication, author A. Brooks Harris was also affiliated with Tel Aviv University, Tel Aviv, \\ Israel. Currently, he is a faculty member in the Physics Department at the University of Pennsylvania.
}




\title{
Magnetic anisotropies and general on-site Coulomb interactions in the cuprates
}

\author{
O. Entin-Wohlman \\ School of Physics and Astronomy, Raymond and Beverly Sackler Faculty of Exact Sciences, Tel Aviv University, Tel Aviv 69978, Israel
}

A. B. Harris

School of Physics and Astronomy, Raymond and Beverly Sackler Faculty of Exact Sciences, Tel Aviv University, Tel Aviv 69978, Israel and Department of Physics, University of Pennsylvania, Philadelphia, Pennsylvania 19104-6369

Amnon Aharony

School of Physics and Astronomy, Raymond and Beverly Sackler Faculty of Exact Sciences, Tel Aviv University, Tel Aviv 69978, Israel

(Received 12 June 1995)

\begin{abstract}
This paper derives the anisotropic superexchange interactions from a Hubbard model for excitations within the copper $3 d$ band and the oxygen $2 p$ band of the undoped insulating cuprates. We extend the recent calculation of Yildirim et al. [Phys. Rev. B 52, 10239 (1995)] in order to include the most general on-site Coulomb interactions (including those which involve more than two orbitals) when two holes occupy the same site. Our general results apply when the oxygen ions surrounding the copper ions form an octahedron which has tetragonal symmetry (but may be rotated as in lanthanum cuprate). For the tetragonal cuprates we obtain an easy-plane anisotropy in good agreement with experimental values. We predict the magnitude of the small in-plane anisotropy gap in the spin-wave spectrum of $\mathrm{YBa}_{2} \mathrm{Cu}_{3} \mathrm{O}_{6}$.
\end{abstract}

\section{INTRODUCTION}

The magnetic anisotropies of the family of compounds with structures similar to that of $\mathrm{La}_{2} \mathrm{CuO}_{4}$ (LCO) has been a subject of much current interest. ${ }^{1-8}$ These materials are antiferromagnets, ${ }^{9}$ with a dominant easy-plane anisotropy. ${ }^{10}$ The parent compound, LCO, is orthorhombic in the regime of interest. In this structure the oxygen octahedra surrounding each copper rotate by a small angle relative to their orientation in the tetragonal phase. This phase was found to have an antisymmetric Dzyaloshinskii-Moriya interaction, as allowed by the lack of inversion symmetry about the center of a $\mathrm{Cu}-\mathrm{Cu}$ bond. ${ }^{11}$ More recently, members of this family which remain tetragonal, such as $\mathrm{Sr}_{2} \mathrm{CuO}_{2} \mathrm{Cl}_{2}$, ${ }^{12}$ have been studied. In many such compounds, for example, $\mathrm{Sr}_{2} \mathrm{CuO}_{2} \mathrm{Cl}_{2},{ }^{13} \mathrm{Nd}_{2} \mathrm{CuO}_{4},{ }^{14}$ and $\mathrm{Pr}_{2} \mathrm{CuO}_{4},{ }^{15}$ the gap in the spin-wave spectrum at zero wave vector due to the easyplane anisotropy has been found to be about $5 \mathrm{meV}$, just as in $\mathrm{LCO}^{16}$

Since these systems involve copper ions in a $3 d^{9}$ configuration which have spin $1 / 2$, the usually dominant mechanism of single-ion anisotropy does not come into play. Instead, anisotropy must be due to the anisotropy of the superexchange interaction. Microscopic derivations of the anisotropy energies have been carried out ${ }^{1-8}$ on the basis of Anderson's theory $^{17}$ of kinetic superexchange, and Moriya's extension ${ }^{18}$ to incorporate spin-orbit interactions. These have been mostly confined to the consideration of the orthorhombic phase and were based on terms requiring the existence of a distortion. As a result, these calculations produce anisotropy energies which are proportional to the distortion angle and which therefore vanish in the tetragonal phase. However, since the experiments cited above ${ }^{13-16}$ indicate that the magnitude of the easy-plane anisotropy is independent of the structural distortion, these theories, while giving correct in- formation about the Dzyaloshinskii-Moriya interaction, ${ }^{18,19}$ did not provide a satisfactory basis for understanding the easy-plane anisotropy. Recognizing this fact, Yildirim et al. ${ }^{6,7}$ undertook an investigation of a model designed to calculate the anisotropy of the superexchange interaction so as to account correctly for the tetragonal symmetry of the lattice. In their treatment all five crystal-field states of the copper $3 d$ band (with a single hole on each copper ion) were taken into account, as well as all the (occupied) $2 p$ states of the oxygen ions. As a result, the exchange interaction associated with each $\mathrm{Cu}-\mathrm{Cu}$ bond had biaxial exchange anisotropy. (I.e., all three diagonal components of the exchange tensor were in general different.) In that work, a model of Coulomb interactions was used which contained more terms than usual, but was still not completely general. Here we carry out the calculation with completely general on-site Coulomb interactions necessary to treat excited states with two holes on a single $\mathrm{Cu}$ ion, or on a single oxygen ion, assuming tetragonal site symmetry. As is known, ${ }^{20}$ such interactions can be parametrized in terms of only three parameters, the Racah parameters, $A, B$, and $C$, with $A \gg B$ and $A \gg C$. In Refs. 6 and 7 it was shown that for tetragonal symmetry the exchange anisotropy vanishes if $B=C=0$. Here we obtain results which include all contributions to the exchange anisotropy correct to first order in both $B$ and $C$. Compared to the previous work, we find an additional contribution which slightly increases the biaxiality of the anisotropy, without sensibly changing the easy-plane anisotropy, which still agrees quite well with experimental results. Under certain approximations, our results can be applied to the orthorhombic phase of LCO. We should emphasize, however, that our aim in this paper is to calculate only the anisotropy of the exchange interaction. This point is discussed at the end of Sec. III.

Briefly, this paper is organized as follows. In Sec. II we 
describe the model for which we calculate the exchange anisotropy. In Sec. III we describe the perturbative calculations, Sec. IV contains specific results for tetragonal symmetry, and in Sec. V we discuss and briefly summarize our results. We will attempt to make this paper self-contained, but readers wishing more details on the approach or the history of this problem are advised to consult Ref. 7, Yildirim, Harris, Aharony, and Entin-Wohlman (YHAE). That reference also contains the details of the spin-wave spectrum which results from a calculation of the exchange anisotropy.

\section{THE MODEL}

We start by considering the ground state of the $\mathrm{CuO}_{2}$ plane, when the kinetic energy is completely neglected. In that case, there is a single $3 d$ hole on each copper ion and the oxygen $2 p$ band is completely full. Since the spin of the $\mathrm{Cu}$ hole is arbitrary, this ground state is $2^{N}$-fold degenerate, where $N$ is the number of $\mathrm{Cu}$ ions. The kinetic superexchange interaction is obtained as the effective interaction within this degenerate manifold when the kinetic energy is treated perturbatively. For this purpose we write the Hamiltonian as

$$
\mathscr{H}=\mathscr{H}_{\mathrm{cu}}+\mathscr{H}_{\mathrm{O}}+\mathscr{H}_{\text {hop }} .
$$

Here, $\mathscr{H}_{\mathrm{Cu}}\left(\mathscr{H}_{\mathrm{O}}\right)$ describes the Hamiltonian of the copper (oxygen) ions and $\mathscr{H}_{\text {hop }}$ is the kinetic energy (hopping between $\mathrm{Cu}$ and $\mathrm{O}$ ions). To describe the $\mathrm{Cu}$ ions we work in a representation in which the crystal-field Hamiltonian is diagonal. Diagonalization of the crystal field potential yields five spatial $d$ states, denoted by $|\alpha\rangle$, with site energies $\epsilon_{\alpha}$. Each such energy is doubly degenerate due to spin $\sigma= \pm 1$. In the systems under consideration, the five $d$-states $|\alpha\rangle$ are determined by the tetragonal symmetry. Even in the orthorhombic phase the site symmetry of the copper ions can be taken to be tetragonal, since the crystal-field is primarily generated by the neighboring oxygen ions, which, to a good approximation, still form an octahedron, albeit a rotated one. For tetragonal symmetry we label these crystal field states as $|0\rangle \sim x^{2}-y^{2}, \quad|1\rangle \sim 3 z^{2}-r^{2}, \quad|z\rangle \sim x y, \quad|x\rangle \sim y z, \quad$ and $|y\rangle \sim z x$, where the $z$ axis is perpendicular to the $\mathrm{CuO}_{2}$ plane and $|0\rangle$ is the lowest energy single-particle state. Thus

$$
\begin{aligned}
\mathscr{H}_{\mathrm{Cu}}= & \sum_{i \alpha \sigma} \epsilon_{\alpha} d_{i \alpha \sigma}^{\dagger} d_{i \alpha \sigma}+\frac{\lambda}{2} \sum_{i \alpha \beta} \sum_{\sigma \sigma^{\prime}} \mathbf{L}_{\alpha \beta} \cdot[\vec{\sigma}]_{\sigma \sigma^{\prime}} d_{i \alpha \sigma}^{\dagger} d_{i \beta \sigma^{\prime}} \\
& +\frac{1}{2} \sum_{\alpha \beta \gamma \delta} \sum_{i \sigma \sigma^{\prime}} U_{\alpha \beta \gamma \delta} d_{i \alpha \sigma}^{\dagger} d_{i \beta \sigma^{\prime}}^{\dagger} d_{i \gamma \sigma^{\prime}} d_{i \delta \sigma}
\end{aligned}
$$

where $d_{i \alpha \sigma}^{\dagger}$ creates a hole in the crystal-field state $\alpha$ at site $i$ with spin $\sigma$. Here the first term is the crystal-field Hamiltonian. The second term is the spin-orbit interaction, where $\lambda$ is the spin-orbit coupling constant and $\mathbf{L}_{\alpha \beta}$ denotes the matrix elements of the orbital angular momentum vector between the crystal-field states $\alpha$ and $\beta$. The last term is the Coulomb interaction, where

$$
\begin{aligned}
U_{\alpha \beta \gamma \delta} & =\int d \mathbf{r}_{1} \int d \mathbf{r}_{2} \psi_{\alpha}\left(\mathbf{r}_{1}\right) \psi_{\beta}\left(\mathbf{r}_{2}\right) \frac{e^{2}}{r_{12}} \psi_{\gamma}\left(\mathbf{r}_{2}\right) \psi_{\delta}\left(\mathbf{r}_{1}\right) \\
& \equiv(\alpha \delta \mid \beta \gamma)
\end{aligned}
$$

in the notation ${ }^{21}$ of Table A26 of Ref. 20. The second term in Eq. (1) gives the Hamiltonian of the oxygen ions. We assume that the spin-orbit interaction on the oxygen is much smaller than that on the copper and may be neglected. Hence we write

$$
\begin{aligned}
\mathscr{H}_{\mathrm{O}}= & \sum_{k n \sigma} \epsilon_{n} p_{k n \sigma}^{\dagger} p_{k n \sigma} \\
& +\frac{1}{2} \sum_{n_{1} n_{2} n_{3} n_{4}} \sum_{\sigma \sigma^{\prime} k} U_{n_{1} n_{2} n_{3} n_{4}} p_{k n_{1} \sigma}^{\dagger} p_{k n_{2} \sigma^{\prime}}^{\dagger} p_{k n_{3} \sigma^{\prime}} p_{k n_{4} \sigma},
\end{aligned}
$$

in which $p_{k n \sigma}^{\dagger}$ creates a hole in one of the three $p$ orbitals (denoted by $n$ ) on the oxygen at site $k$. Here the Coulomb matrix element is obtained analogously to Eqs. (3). Finally, $\mathscr{H}_{\text {hop }}$ describes the hopping between two neighboring oxygen and copper ions:

$$
\mathscr{H}_{\mathrm{hop}}=\sum_{i \alpha \sigma} \sum_{k n \sigma} t_{\alpha n}^{i k} d_{i \alpha \sigma}^{\dagger} p_{k n \sigma}+\text { H.c., }
$$

in which $t_{\alpha n}^{i k}$ is the hopping matrix element and H.c. denotes the Hermitian conjugate of the preceding terms. As mentioned, it is $\mathscr{H}_{\text {hop }}$ that lifts the degeneracy of the $2^{N}$-fold degenerate ground state.

In order to derive the effective magnetic Hamiltonian it is convenient to start from the unperturbed Hamiltonian which contains the spin-orbit interaction exactly. To achieve this, we introduce the unitary transformation which diagonalizes the single-particle part of $\mathscr{H}_{\mathrm{Cu}}$. As noted previously, ${ }^{6,7}$ the spin dependence of this transformation is fixed by tetragonal symmetry, so that we can write

$$
d_{i \alpha \sigma}=\sum_{a \sigma^{\prime}} m_{\alpha a}[\sigma(\alpha)]_{\sigma \sigma^{\prime}} c_{i a \sigma^{\prime}}
$$

where $c_{i a \sigma^{\prime}}$ destroys a hole in the exact eigenstate of the Hamiltonian which consists of the crystal-field and spin-orbit interactions. These states have a site label $i$, a state label (for which we use roman letters), and a pseudospin index $\sigma^{\prime}$. In Eq. (6) we define $\sigma(\alpha)$ for each crystal-field state $|\alpha\rangle$ as follows: $\sigma(\alpha)=\sigma_{\alpha}$ is the Pauli matrix for $\alpha=x, y, z$ and $\sigma(\alpha)=\mathscr{T}$ is the unit matrix, for $\alpha=0,1$. The $5 \times 5$ matrix $\mathbf{m}$ is the solution to

$$
\epsilon_{\alpha} m_{\alpha b}+\sum_{\beta} \mathscr{C}_{\alpha \beta} m_{\beta b}=E_{b} m_{\alpha b}
$$

with $\Sigma_{\alpha} m_{\alpha a}^{*} m_{\alpha b}=\delta_{a b}$, where $\delta$ is the Kronecker delta function. Here $\mathscr{C}_{\alpha \beta}$ are related to the matrix elements of the orbital angular momentum vector and are given by

$$
\begin{gathered}
\mathscr{L}_{0 z}=-i \lambda, \quad \mathscr{L}_{0 x}=\mathscr{L}_{0 y}=i \lambda / 2, \quad \mathscr{L}_{1 x}=-\mathscr{L}_{1 y}=i \sqrt{3} \lambda / 2, \\
\mathscr{L}_{z x}=\mathscr{L}_{z y}=\mathscr{L}_{x y}=\lambda / 2 .
\end{gathered}
$$

Matrix elements not listed and not obtainable using $\mathscr{C}_{\alpha \beta}=\mathscr{L}_{\beta \alpha}^{*}$ are zero. When $\lambda \rightarrow 0$, each state $|a\rangle$ approaches one of the states $|\alpha\rangle$. Using this identification, the indices 
TABLE I. Values (in eV) of the parameters used to calculate the anisotropic exchange. For a discussion of these values, see YHAE.

\begin{tabular}{lcccccccccc}
\hline \hline$\lambda$ & $A$ & $B$ & $C$ & $(p d \sigma)$ & $\epsilon_{1}$ & $\epsilon_{x}=\epsilon_{y}$ & $\epsilon_{z}$ & $\epsilon_{p_{x}}$ & $\epsilon_{p_{y}}$ & $\epsilon_{p_{z}}$ \\
0.1 & 7.0 & 0.15 & 0.58 & 1.5 & 1.8 & 1.8 & 1.8 & 3.25 & 3.25 & 3.25 \\
\hline
\end{tabular}

$a$ also run over the values $0,1, z, x$, and $y$. We use the values of the parameters which are listed in Table I and are discussed in detail in YHAE.

\section{PERTURBATION EXPANSION}

We now divide the total Hamiltonian $\mathscr{H}$ into an unperturbed part, $\mathscr{H}_{0}$, and a perturbation term $\mathscr{H}_{1}$. The part $\mathscr{H}_{0}$ contains the single-particle Hamiltonians on the coppers and on the oxygens, and the leading on-site Coulomb potentials,

$$
\begin{aligned}
\mathscr{H}_{0}= & \sum_{i a \sigma} E_{a} c_{i a \sigma}^{\dagger} c_{i a \sigma}+\frac{U_{0}}{2} \sum_{i a b} c_{i a \sigma^{\prime}}^{\dagger} c_{i b \sigma^{\prime}}^{\dagger} c_{i b \sigma^{\prime}} c_{i a \sigma} \\
& +\sum_{k n \sigma} \epsilon_{n} p_{k n \sigma}^{\dagger} p_{k n \sigma}+\frac{U_{P}}{2} \sum_{\substack{k n n^{\prime} \\
\sigma \sigma^{\prime}}} p_{k n \sigma}^{\dagger} p_{k n^{\prime} \sigma^{\prime}}^{\dagger} p_{k n^{\prime} \sigma^{\prime}} p_{k n \sigma}
\end{aligned}
$$

For tetragonal site symmetry, we choose $U_{0} \equiv U_{\alpha \alpha \alpha \alpha}$ $=A+4 B+C$ and $U_{P} \equiv U_{n n n n}$. The perturbation Hamiltonian includes the kinetic energy and the remaining Coulomb interactions. Because of the transformation (6), the hopping becomes spin dependent. Thus we have

$$
\mathscr{H}_{1}=\mathscr{H}_{\mathrm{hop}}+\Delta \mathscr{H}_{\mathrm{C}},
$$

in which

$$
\mathscr{H}_{\mathrm{hop}}=\sum_{i a \sigma} \sum_{k n \sigma^{\prime}}\left(\vec{t}_{a n}^{i k}\right)_{\sigma^{\prime} \sigma^{\prime}} c_{i a \sigma^{\prime}}^{\dagger} p_{k n \sigma}+\text { H.c., }
$$

with the $2 \times 2$ matrix

$$
\widetilde{t}_{a n}^{i k}=\sum_{\alpha} t_{\alpha n}^{i k} m_{\alpha a}^{*} \sigma(\alpha)
$$

and $\Delta \mathscr{H}_{C}$ describes the perturbation parts of the on-site Coulomb potentials,

$$
\begin{aligned}
\Delta \mathscr{H}_{C}= & \frac{1}{2} \sum_{\begin{array}{c}
s s^{\prime} s_{1} s_{1}^{\prime} \\
i a b c d
\end{array}} \Delta \bar{U}_{s s^{\prime} s_{1} s_{1}^{\prime}}(\text { abcd }) c_{i a s}^{\dagger} c_{i b s^{\prime}}^{\dagger} c_{i c s_{1}} c_{i d s_{1}^{\prime}} \\
& +\frac{1}{2} \sum_{\substack{k \sigma \sigma^{\prime} \\
n_{1} n_{2} n_{3} n_{4}}} \Delta U_{n_{1} n_{2} n_{3} n_{4}} p_{k n_{1} \sigma}^{\dagger} p_{k n_{2} \sigma^{\prime}}^{\dagger} p_{k n_{3} \sigma^{\prime}} p_{k n_{4} \sigma},
\end{aligned}
$$

with

$$
\begin{aligned}
\Delta \bar{U}_{s s^{\prime} s_{1} s_{1}^{\prime}}(\text { abcd })= & \sum_{\alpha \beta \gamma \delta} \Delta U_{\alpha \beta \gamma \delta} m_{\alpha a^{*}}^{*} m_{\beta b}^{*} m_{\gamma c} m_{\delta d} \\
& \times[\sigma(\alpha) \sigma(\delta)]_{s s_{1}^{\prime}}[\sigma(\beta) \sigma(\gamma)]_{s^{\prime} s_{1}} .
\end{aligned}
$$

$\Delta \mathbf{U}$ involves only the small Racah coefficients, $B$ and $C$.

The effective magnetic interaction $\mathscr{H}(i, j)$ between magnetic ions $i$ and $j$ is found by perturbation expansion with respect to $\mathscr{H}_{1}$. All the perturbation contributions to $\mathscr{H}(i, j)$ should involve products of matrix elements which begin and end within the $2^{N}$-fold degenerate ground-state manifold of $\mathscr{H}_{0}$, each state of which has one hole at each copper site, with arbitrary spin $\sigma$. Denoting such a ground state by $\left|\psi_{0}\right\rangle$, and concentrating on perturbation terms which involve only two coppers $i$ and $j$ and one oxygen between them, it is convenient to replace $\left|\psi_{0}\right\rangle$ by $\sum_{\sigma \sigma_{1}} c_{i 0 \sigma}^{\dagger} c_{j 0 \sigma_{1}}^{\dagger} c_{j 0 \sigma_{1}} c_{i 0 \sigma}\left|\psi_{0}\right\rangle$. This ensures that $\left|\psi_{0}\right\rangle$ indeed has exactly one hole on each copper $i$ and $j$. Clearly the lowest-order contributions to the energy are of order $\vec{t}^{4}$. There are two possible channels in this order, which we denote by $a$ and $b$. In channel $a$, the hole is transferred from one of the coppers to the oxygen, then to the second copper. Afterwards, one of the holes on this second copper returns to the empty copper. Hence in this channel there are two holes on the copper in an intermediate state. In channel $b$, the hole is transferred from one of the coppers to the oxygen, and then a second hole is taken from another copper to the same oxygen. Then the two holes hop back, each to one of the initial coppers. Thus in channel $b$ there are two holes on the oxygen in an intermediate state. When the perturbation contributions coming from the Coulomb potential $\Delta \mathscr{H}_{C}$ [Eqs. (10), (13) and (14)] are included, the on-site interactions on the copper are effective in channel $a$, and those on the oxygen appear in channel $b$. These contributions are of order $t^{-4} \Delta \mathscr{H}_{C}$.

The perturbation contributions to order $\widetilde{t}^{4}$ are the same as those given by YHAE and are

$$
\mathscr{H}^{(1)}(i, j)=\sum_{n n^{\prime}} g_{n n^{\prime}} \operatorname{Tr}\left\{\vec{\sigma} \cdot \mathbf{S}_{i} \vec{t}_{0 n}^{i k} \vec{t}_{n 0}^{k j} \vec{\sigma} \cdot \mathbf{S}_{j} \vec{t}_{0 n^{\prime}}^{j k} \vec{t}_{n^{\prime} 0}^{k i}\right\}
$$

in which

$$
\mathbf{S}_{i}=\frac{1}{2} \sum_{\sigma \sigma^{\prime}} c_{i 0 \sigma}^{\dagger}(\vec{\sigma})_{\sigma \sigma^{\prime}} c_{i 0 \sigma^{\prime}}
$$

is the spin on the copper at site $i$ in the orbital state $|0\rangle$, $\widetilde{t}_{0 n}^{i k}$ is the $2 \times 2$ matrix given by Eq. (12) and

$$
g_{n n^{\prime}}=\frac{2}{U_{0}} \frac{1}{\epsilon_{n} \epsilon_{n^{\prime}}}+\frac{1}{U_{P}+\epsilon_{n}+\epsilon_{n^{\prime}}}\left(\frac{1}{\epsilon_{n}}+\frac{1}{\epsilon_{n^{\prime}}}\right)^{2} .
$$

The first term in $g_{n n^{\prime}}$ results from channel $a$, while the second arises from channel $b$. In deriving expression (15) we have assumed that the single-particle energy $E_{a=0}$ on the copper is equal to zero.

From the form of $\mathscr{H}^{(1)}(i, j)$ it is clear that it may yield anisotropic magnetic interactions only when the effective hopping between the coppers, $\widetilde{t}^{\imath} \tilde{t}^{k j}$, involves spin flips. 
When the hopping conserves the spin, the $\tilde{t}$ products are proportional to the $2 \times 2$ unit matrix and the trace in Eq. (15) gives a result proportional to $\mathbf{S}_{i} \cdot \mathbf{S}_{j}$. This is indeed the case for tetragonal symmetry, as we discuss in the next section. ${ }^{6,7}$ In the orthorhombic phase of $\mathrm{La}_{2} \mathrm{CuO}_{4}$, however, the effective hopping between the coppers is accompanied by spin flip, and consequently $\mathscr{H}^{(1)}(i, j)$ includes the antisymmetric
Dzyaloshinskii-Moriya interaction, as well as symmetric magnetic anisotropies. . $^{2,3}$

We now turn to the contributions of the Coulomb potential. In particular, we consider contributions to the magnetic energy which are of order $\tilde{t}^{4}$ and first order in $\Delta \mathscr{H}_{C}$. Following the approach of YHAE one finds that the processes from channel $a$ (indicated by a subscript " $a$ ") yield

$$
\begin{aligned}
& \mathscr{H}_{a}^{(2)}(i, j)=\left[\sum _ { \substack { a , b , \neq 0 \\
\alpha \beta \gamma \delta } } \frac { \Delta U _ { \alpha \beta \gamma \delta } } { ( U _ { 0 } + E _ { a } ) ( U _ { 0 } + E _ { b } ) } \left(\operatorname{Tr}\left\{\vec{\sigma} \cdot \mathbf{S}_{i} \sum_{n} \frac{\vec{t}_{0 n}^{i k} \vec{t}_{n b}^{k j}}{\epsilon_{n}} \sigma(\alpha) \sigma(\delta) \sum_{n^{\prime}} \frac{\vec{t}_{a n^{\prime}}^{j^{k}} \vec{t}_{n^{\prime} 0}^{\star i}}{\epsilon_{n^{\prime}}}\right\} \operatorname{Tr}\left\{\vec{\sigma} \cdot \mathbf{S}_{j} \sigma(\beta) \sigma(\gamma)\right\} m_{\alpha b}^{*} m_{\beta 0}^{*} m_{\gamma 0} m_{\delta a}\right.\right. \\
& \left.\left.-\operatorname{Tr}\left\{\vec{\sigma} \cdot \mathbf{S}_{i} \sum_{n} \frac{\vec{t}_{0 n}^{i k} \vec{t}_{n b}^{k j}}{\epsilon_{n}} \sigma(\alpha) \sigma(\delta) \vec{\sigma} \cdot \mathbf{S}_{j} \sigma(\beta) \sigma(\gamma) \sum_{n^{\prime}} \frac{\vec{t}_{a n^{\prime}}^{j^{k}} \tau_{n^{\prime} 0}^{\star i}}{\epsilon_{n^{\prime}}}\right\} m_{\alpha b}^{*} m_{\beta 0}^{*} m_{\gamma a} m_{\delta 0}\right)+(i \leftrightarrow j)\right],
\end{aligned}
$$

where $i \leftrightarrow j$ denotes the sum of previous terms with $i$ and $j$ interchanged. Here it is convenient to classify the Coulomb matrix elements into two classes, by writing

$$
\Delta U_{\alpha \beta \gamma \delta}=U_{\alpha \beta \gamma \delta}^{(1)}+U_{\alpha \beta \gamma \delta}^{(2)},
$$

where $U_{\alpha \beta \gamma \delta}^{(1)}$ is nonzero only if

$$
\sigma(\alpha)=\sigma(\delta), \quad \text { and } \quad \sigma(\beta)=\sigma(\gamma),
$$

while $U_{\alpha \beta \gamma \delta}^{(2)}$ is nonzero only if

$$
\sigma(\alpha) \sigma(\delta)=C_{1} \sigma_{\mu}=C_{2} \sigma(\beta) \sigma(\gamma), \quad \text { for } \mu=x, y, z,
$$

where $C_{1}$ and $C_{2}$ are constants which may be imaginary. This classification will be of immediate use. For the elements denoted $U_{\alpha \beta \gamma \delta}^{(1)}$, the products $\sigma(\alpha) \sigma(\delta)$ and $\sigma(\beta) \sigma(\gamma)$ are both proportional to the unit matrix. Therefore the first term in the square brackets of Eq. (18) vanishes, and one is left with the second term alone. For the matrix elements denoted by $U_{\alpha \beta \gamma \delta}^{(2)}$, both products $\sigma(\alpha) \sigma(\delta)$ and $\sigma(\beta) \sigma(\gamma)$ are proportional to $\sigma_{\mu}, \mu=x, y, z$. In treating this contribution it is convenient to use the identity

$$
\sigma_{\mu} \vec{\sigma} \cdot \mathbf{S} \sigma_{\mu}=2 \sigma_{\mu} S_{\mu}-\vec{\sigma} \cdot \mathbf{S}
$$

We see that the matrix elements $U_{\alpha \beta \gamma \delta}^{(2)}$ give rise to magnetic interactions which depend on the Cartesian index $\mu$. Rearranging the terms in (18) and defining

$$
Q_{a b}=\sum_{\alpha \beta \gamma \delta} \frac{U_{\alpha \beta \gamma \delta}^{(2)}-U_{\alpha \beta \gamma \delta}^{(1)}}{\left(U_{0}+E_{a}\right)\left(U_{0}+E_{b}\right)} m_{\alpha b}^{*} m_{\beta 0}^{*} m_{\gamma 0} m_{\delta a},
$$

$$
\begin{aligned}
K_{a b}^{\mu}= & \sum_{\alpha \beta \gamma \delta} \frac{U_{\alpha \beta \gamma \delta}^{(2)}}{\left(U_{0}+E_{a}\right)\left(U_{0}+E_{b}\right)}\left(m_{\alpha b}^{*} m_{\beta 0}^{*}-m_{\beta b}^{*} m_{\alpha 0}^{*}\right) \\
& \times\left(m_{\delta a} m_{\gamma 0}-m_{\delta 0} m_{\gamma a}\right),
\end{aligned}
$$

we obtain

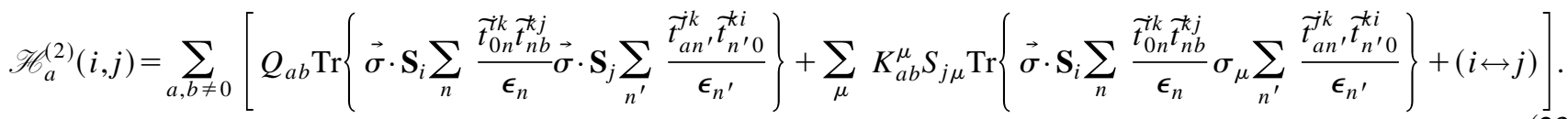

Note that the sum over state labels in Eqs. (22a) and (22b) is restricted by the conditions of Eqs. (20a) and (20b). One notes that the first term in Eq. (23) has a structure similar to that of $\mathscr{H}^{(1)}(i, j)$ in Eq. (15) and therefore has the same magnetic symmetries. The second term, however, leads to magnetic anisotropy even for tetragonal symmetry, for which the effective hopping between the coppers is spin independent. This is elaborated upon in the next section.

Finally we consider the processes in channel $b$, in which the two holes are on the oxygen in an intermediate state and thus experience the Coulomb interaction on the oxygen. These processes yield

$$
\begin{aligned}
& \mathscr{H}_{b}^{(2)}(i, j)=\sum_{n n^{\prime} n_{1} n_{1}^{\prime}}\left(\frac{1}{\epsilon_{n}}+\frac{1}{\epsilon_{n^{\prime}}}\right)\left(\frac{1}{\epsilon_{n_{1}}}+\frac{1}{\epsilon_{n_{1}^{\prime}}}\right) \frac{\Delta U_{n_{1} n_{1}^{\prime} n^{\prime}}}{\left(U_{P}+\epsilon_{n}+\epsilon_{n^{\prime}}\right)\left(U_{P}+\epsilon_{n_{1}}+\epsilon_{n_{1}^{\prime}}\right)}\left[\operatorname{Tr}\left\{\vec{\sigma} \cdot \mathbf{S}_{i} \tilde{t}_{0 n_{1}}^{i k} \vec{t}_{n^{\prime} 0}^{k i}\right\} \operatorname{Tr}\left\{\vec{\sigma} \cdot \mathbf{S}_{j} \vec{t}_{0 n_{1}^{\prime} k}^{\tau_{1}^{k j}} \vec{t}_{n 0}\right\}\right. \\
& \left.-\operatorname{Tr}\left\{\vec{\sigma} \cdot \mathbf{S}_{i} \vec{t}_{0 n_{1}}^{i k} \vec{t}_{n^{\prime} 0}^{k j} \vec{\sigma} \cdot \mathbf{S}_{j} \vec{t}_{0 n_{1}^{j}}^{j k} \vec{t}_{n 0}^{k i}\right\}\right]
\end{aligned}
$$


To the order we work, the total magnetic interaction between a pair of copper ions is given by

$$
\mathscr{H}(i, j)=\mathscr{H}^{(1)}(i, j)+\mathscr{H}_{a}^{(2)}(i, j)+\mathscr{H}_{b}^{(2)}(i, j) .
$$

In the next section we examine $\mathscr{H}(i, j)$ in the tetragonal phase. In the orthorhombic phase the situation is more complicated. In principle, one should start by replacing the tetragonal crystal-field states by those which are calculated in the presence of the orthorhombic distortion. A reasonable approximation is to take account of this distortion by considering the crystal-field states of the octahedron of oxygen ions assuming this octahedron to be rotated rigidly away from its orientation in the tetragonal phase. Then, to the extent that the crystal field is wholly determined by the octahedron of oxygen neighbors, it will be tetragonal in the rotated coordinate system fixed by the shell of oxygen neighbors. Then the result of Eq. (25) can be used. The major complication is that the hopping matrix elements are those between rotated orbitals, as will be detailed elsewhere. ${ }^{22}$

Some comments concerning the applicability of our results should be made. As discussed in YHAE, we believe that the use of perturbation theory is justified for the calculation of the anisotropy of the superexchange Hamiltonian. In contrast, for the isotropic terms it has been shown ${ }^{23}$ that perturbation theory is not reliable, mainly because the energies of the excited states of the oxygen levels are not very large in comparison to $t$. However, since the spin-orbit interaction takes place on the copper ions, this effect is much reduced in the anisotropic terms. In addition, there are contributions to the isotropic interactions which cannot be obtained by consideration of a single bond. In one of these, a hole hops from a copper to one nearest-neighboring oxygen, then diagonally to a different nearest-neighbor oxygen, and finally back to the original copper. ${ }^{23}$ Also, the effective spin Hamiltonian acquires isotropic contributions from processes of order $\vec{t}^{8}$ which involve a hole hopping around a plaquette of $\mathrm{Cu}$ ions. ${ }^{24}$ These plaquette interactions involve both twospin interactions (between nearest- and next-nearest neighbors) and four-spin interactions.

\section{TETRAGONAL SYMMETRY}

Here we study the effective magnetic Hamiltonian $\mathscr{H}(i, j)$ [Eqs. (15) and (23)-(25)] for the case of tetragonal symmetry. For this symmetry the effective interaction which is bilinear in the spin operators must be of the form

$$
\mathscr{H}(i, j)=\sum_{\mu} J_{\mu \mu}(i, j) S_{\mu}(i) S_{\mu}(j)
$$

where $\mu=x, y, z$. Since we are interested in the anisotropic part of the exchange interaction, we will drop any contributions which we identify as being isotropic. Obviously, our results cannot be used for the magnitude of an individual $J_{\mu \mu}$, but rather apply to the difference between two such quantities.
Investigation of Eqs. (15), (23), and (24) reveals that one can define an effective hopping between the copper ions, which is generally given by the product $\widetilde{t}_{a n}^{i k} \tilde{t}_{n^{\prime} b}^{k j}$. \{The interaction $\mathscr{H}_{b}^{(2)}(i, j)[$ Eq. (24)] requires also the cases $i=j$. $\}$ We therefore start by examining this quantity. To this end we note that the nonzero hopping matrix elements between the tetragonal states $|\alpha\rangle$ on the coppers and the states $|n\rangle$ on the oxygen are $t_{0 p_{x}}, t_{1 p_{x}}, t_{y p_{z}}$, and $t_{z p_{y}}$ for a bond along the $x$ direction in the $\mathrm{CuO}_{2}$ plane, and analogously $t_{0 p_{y}}, t_{1 p_{y}}$, $t_{x p_{z}}$, and $t_{z p_{x}}$ for a bond along $y .^{6,7}$ Using now Eq. (6) we find

$$
\widetilde{t}_{a n} \widetilde{t}_{n^{\prime} b}=\sum_{\alpha \beta} t_{\alpha n} t_{n^{\prime} \beta} m_{\alpha a}^{*} m_{\beta b} \sigma(\alpha) \sigma(\beta),
$$

where we have omitted the site indices for convenience. It therefore follows that in the case $n=n^{\prime}$ the quantity in Eq. (27) is proportional to the $2 \times 2$ unit matrix, namely, the effective hopping between the copper ions is not accompanied by spin flip. This means that in the expressions for the interactions $\mathscr{H}^{(1)}(i, j)$ and $\mathscr{H}_{a}^{(2)}(i, j)$ [Eqs. (15) and (23)] we may take all $\widetilde{t}$ 's outside the trace. Consequently, the contribution to the magnetic energy from Eq. (15) is isotropic, and so is that from the first term in Eq. (23), proportional to $Q_{a b}$. The second term in $\mathscr{H}_{a}^{(2)}(i, j)$, which arises from $U_{\alpha \beta \gamma \delta}^{(2)}$, leads to an anisotropic magnetic interaction of the form of Eq. (26), with

$$
J_{\mu \mu}=4 \sum_{a, b \neq 0} K_{a b}^{\mu} \sum_{n} \frac{\widetilde{t}_{0 n} \widetilde{t}_{n b}}{\epsilon_{n}} \sum_{n^{\prime}} \frac{\widetilde{t}_{a n^{\prime}} \widetilde{t}_{n^{\prime} 0}}{\epsilon_{n^{\prime}}} .
$$

We now consider the magnetic symmetry of $\mathscr{H}_{b}^{(2)}(i, j)$, which results from the on-site Coulomb potential on the oxygen. The only nonzero matrix elements $\Delta U_{n_{1} n_{1}^{\prime} n n^{\prime}} \operatorname{are}^{25}$

$$
\Delta U_{n n^{\prime} n^{\prime} n}, \quad \Delta U_{n n^{\prime} n n^{\prime}}, \quad \Delta U_{n n n^{\prime} n^{\prime}}
$$

The first of these implies terms of the form ${\widetilde{t_{0 n}}}_{t_{n 0}}$ [cf. Eq. (24)], which are proportional to the unit matrix. As a result the first term in the square brackets of (24) disappears. The second, which yields an isotropic interaction, can be combined into $\mathscr{H}^{(1)}(i, j)$ by redefining $g_{n n^{\prime}}[$ Eq. (17)] to be

$$
g_{n n^{\prime}}=\frac{2}{U_{0}} \frac{1}{\epsilon_{n} \epsilon_{n^{\prime}}}+\frac{1}{U_{P}+\Delta U_{n n^{\prime} n^{\prime} n}+\epsilon_{n}+\epsilon_{n^{\prime}}}\left(\frac{1}{\epsilon_{n}}+\frac{1}{\epsilon_{n^{\prime}}}\right)^{2} .
$$

For simplicity we have put the $\Delta U$ in the denominator of this expression. The results of this paper are correct to first order in $\Delta \mathscr{H}_{c}$.

The other two matrix elements of Eq. (29) lead to 


$$
\begin{aligned}
\mathscr{H}_{b}^{(2)}(i, j)= & \sum_{\substack{n n^{\prime} \\
n \neq n^{\prime}}}\left[\Delta U_{n n^{\prime} n n^{\prime}}\left(\frac{1}{\epsilon_{n}}+\frac{1}{\epsilon_{n^{\prime}}}\right)^{2}\left(\frac{1}{U_{P}+\epsilon_{n}+\epsilon_{n^{\prime}}}\right)^{2}\left(\operatorname{Tr}\left\{\vec{\sigma} \cdot \mathbf{S}_{i} \widetilde{t}_{0 n} \widetilde{t}_{n^{\prime} 0}\right\} \operatorname{Tr}\left\{\vec{\sigma} \cdot \mathbf{S}_{j} \widetilde{t}_{0 n^{\prime}}, \widetilde{t}_{n 0}\right\}-\operatorname{Tr}\left\{\vec{\sigma} \cdot \mathbf{S}_{i} \widetilde{t_{0 n}} \widetilde{t_{n^{\prime} 0}} \vec{\sigma} \cdot \mathbf{S}_{j} \widetilde{t}_{0 n^{\prime}}, \widetilde{t}_{n 0}\right\}\right)\right. \\
& \left.+\Delta U_{n n^{\prime} n n^{\prime}} \frac{4}{\epsilon_{n} \epsilon_{n^{\prime}}} \frac{1}{\left(U_{P}+2 \epsilon_{n}\right)\left(U_{P}+2 \epsilon_{n^{\prime}}\right)}\left(\operatorname{Tr}\left\{\vec{\sigma} \cdot \mathbf{S}_{i} \widetilde{t}_{0 n} \widetilde{t}_{n^{\prime} 0}\right\} \operatorname{Tr}\left\{\overrightarrow{\boldsymbol{\sigma}} \cdot \mathbf{S}_{j} \widetilde{t}_{0 n} \widetilde{t}_{n^{\prime} 0}\right\}-\operatorname{Tr}\left\{\vec{\sigma} \cdot \mathbf{S}_{i} \widetilde{t}_{0 n} \widetilde{t}_{n^{\prime} 0} \vec{\sigma} \cdot \mathbf{S}_{j} \widetilde{t}_{0 n} \widetilde{t}_{n^{\prime} 0}\right\}\right)\right] .
\end{aligned}
$$

The main point to note here is that although $\widetilde{t_{0 n}} \widetilde{t_{n^{\prime} 0}}$ implies a spin flip in the sense that this product is proportional to one of the Pauli matrices [cf. Eq. (27)], it is the same Pauli matrix for both products appearing in each term of Eq. (31). This makes the resulting interaction isotropic. For example, writing $\widetilde{t_{0 n}}{\widetilde{t_{n}}}_{n^{\prime}} \propto \sigma_{\mu}$, with $\mu=x, y$ or $z$, we find that the spin dependence in each of the terms in Eq. (31) is proportional to

$$
\operatorname{Tr}\left\{\vec{\sigma} \cdot \mathbf{S}_{i} \sigma_{\mu}\right\} \operatorname{Tr}\left\{\vec{\sigma} \cdot \mathbf{S}_{j} \sigma_{\mu}\right\}-\operatorname{Tr}\left\{\vec{\sigma} \cdot \mathbf{S}_{i} \sigma_{\mu} \vec{\sigma} \cdot \mathbf{S}_{j} \sigma_{\mu}\right\}=2 \mathbf{S}_{i} \cdot \mathbf{S}_{j}
$$

where we have used the identity (21). Thus the perturbation processes for which there are two holes on the oxygen in the intermediate state do not contribute to the magnetic anisotropy in tetragonal symmetry. This result holds because the spin-orbit interaction on the oxygen has been discarded.

The anisotropic exchange interactions in the tetragonal phase thus come exclusively from channel $a$. Also, as previously noted, only the term proportional to $\mathbf{K}$ in Eq. (23) gives rise to anisotropy. Furthermore one notes that there is no anisotropic contribution to $J_{\mu \mu}$ from the matrix elements $U_{\alpha \beta \gamma \delta}^{(2)}$ for which $\alpha=\beta$ or $\gamma=\delta$.

Up to now, our results have been valid to all orders in the spin-orbit coupling, $\lambda$. We now obtain an explicit expression for the anisotropic part of $J_{\mu \mu}$ to leading order in $\lambda$, which turns out to be $\mathscr{Q}\left(\lambda^{2}\right)$. To this end we use Eq. (7), from which we get

$$
m_{\alpha a}=\left\{\begin{array}{l}
1 \text { for } \alpha=a \\
\frac{\mathscr{L}_{\alpha a}}{\epsilon_{a}-\epsilon_{\alpha}} \quad \text { for } \alpha \neq a
\end{array}+\mathscr{Q}\left(\lambda^{2}\right),\right.
$$

where $\mathscr{L}_{\alpha a}$ for $\alpha \neq a$ [see Eqs. (8)] is of order $\lambda$. Next we note that the summation indices $a$ and $b$ of Eq. (28) can take the values $|a\rangle,|b\rangle=|1\rangle$ or $|a\rangle,|b\rangle=|x\rangle,|y\rangle$ or $|z\rangle$. We therefore need to evaluate $\Sigma_{n} \widetilde{t}_{0 n} \widetilde{t}_{n b} / \epsilon_{n}$ for $|b\rangle=|1\rangle$ and for $|b\rangle=|\nu\rangle$, where $\nu=x, y, z$. Using Eqs. (12) and (33) we find

$$
\begin{gathered}
\sum_{n} \frac{\widetilde{t_{0 n}} \widetilde{t}_{n 1}}{\epsilon_{n}}=\sum_{n} \frac{t_{0 n} t_{n 1}}{\epsilon_{n}}+\mathscr{O}\left(\lambda^{2}\right), \\
\sum_{n} \frac{\widetilde{t_{0 n}} \widetilde{t}_{n \mu}}{\epsilon_{n}}=\sum_{n} \frac{t_{0 n}^{2}-t_{\mu n}^{2}}{\epsilon_{n}} \frac{\mathscr{L}_{0 \mu}}{\epsilon_{\mu}}+\sum_{n} \frac{t_{0 n} t_{n 1}}{\epsilon_{n}} \frac{\mathscr{B}_{1 \mu}}{\epsilon_{\mu}-\epsilon_{1}} \\
+\mathscr{O}\left(\lambda^{2}\right),
\end{gathered}
$$

where the $t$ 's are the hopping matrix elements for the tetragonal states. Equations (33) and (34), in conjunction with Eq. (22b), imply that the contributions of $U_{\alpha \beta \gamma \delta}^{(2)}$ where more than two of the indices $\alpha, \beta, \gamma$, and $\delta$ take the values $x$, $y$ or $z$ are at least of order $\lambda^{3}$. Take for example the element $U_{1 z x y}^{(2)}$. From Eqs. (22b) and (33) we see that the $m$ products in (22b) are of order $\lambda^{2}$ provided that $|b\rangle=|1\rangle$ and $|a\rangle=|x\rangle$ or $|y\rangle$. In all other cases the $m$ products are at least of order $\lambda^{3}$. But with this choice [see Eqs. (34)] the hopping matrix elements provide another factor of $\lambda$, to render $J_{\mu \mu}$ to be of order $\lambda^{3}$. It follows that out of all nonzero $U_{\alpha \beta \gamma \delta}^{(2)}$, the ones that contribute to $J_{\mu \mu}$ up to order $\lambda^{2}$ are

$$
\begin{gathered}
U_{0 \mu 0 \mu}^{(2)}=U_{\mu 0 \mu 0}^{(2)}, \quad U_{1 \mu 1 \mu}^{(2)}=U_{\mu 1 \mu 1}^{(2)}, \\
U_{0 \mu 1 \mu}^{(2)}=U_{\mu 0 \mu 1}^{(2)}=U_{\mu 1 \mu 0}^{(2)}=U_{1 \mu 0 \mu}^{(2)} .
\end{gathered}
$$

We are now in position to calculate $K_{a b}^{\mu}$. When both $|a\rangle$ and $|b\rangle$ are equal to $|1\rangle$ one finds

$$
\begin{aligned}
K_{11}^{\mu}= & \frac{2}{\left(U_{0}+\epsilon_{1}\right)^{2}}\left[-\frac{\mathscr{L}_{\mu 1} \mathscr{D}_{1 \mu}}{\left(\epsilon_{1}-\epsilon_{\mu}\right)^{2}} U_{0 \mu 0 \mu}^{(2)}-\frac{\mathscr{B}_{\mu 0} \mathscr{L}_{0 \mu}}{\epsilon_{\mu}^{2}} U_{1 \mu 1 \mu}^{(2)}\right. \\
& \left.-\frac{\mathscr{B}_{1 \mu} \mathscr{C}_{\mu 0}+\mathscr{C}_{0 \mu} \mathscr{C}_{\mu 1}}{\epsilon_{\mu}\left(\epsilon_{1}-\epsilon_{\mu}\right)} U_{0 \mu 1 \mu}^{(2)}\right]
\end{aligned}
$$

When $|a\rangle=|1\rangle$ and $|b\rangle=|\mu\rangle$ or vice versa, we have

$$
\begin{aligned}
K_{1 \mu}^{\mu}=\left(K_{\mu 1}^{\mu}\right)^{*}= & \frac{2}{\left(U_{0}+\epsilon_{1}\right)\left(U_{0}+\epsilon_{\mu}\right)}\left[-\frac{\mathscr{L}_{\mu 1}}{\epsilon_{1}-\epsilon_{\mu}} U_{0 \mu 0 \mu}^{(2)}\right. \\
& \left.-\frac{\mathscr{B}_{\mu 0}}{\epsilon_{\mu}} U_{0 \mu 1 \mu}^{(2)}\right]
\end{aligned}
$$

where we have kept terms up to order $\lambda$ since the hopping elements in this case will contribute another factor of $\lambda$. Finally, the case where both $|a\rangle$ and $|b\rangle$ are equal to $|\mu\rangle$ requires terms to order $\lambda^{0}$ and is therefore

$$
K_{\mu \mu}^{\mu}=-\frac{2}{\left(U_{0}+\epsilon_{\mu}\right)^{2}} U_{0 \mu 0 \mu}^{(2)} .
$$

Combining the results of Eqs. (34)-(38) we obtain

$$
\begin{aligned}
J_{\mu \mu}= & -\frac{8 U_{0 \mu 0 \mu}^{(2)}}{\left(U_{0}+\epsilon_{\mu}\right)^{2}}\left|\frac{\mathscr{L}_{\mu 1}}{U_{0}+\epsilon_{1}} \sum_{n} \frac{t_{0 n} t_{n 1}}{\epsilon_{n}}-\frac{\mathscr{L}_{\mu 0}}{\epsilon_{\mu}} \sum_{n} \frac{t_{0 n}^{2}-t_{\mu n}^{2}}{\epsilon_{n}}\right|^{2} \\
& -\frac{8 U_{1 \mu 1 \mu}^{(2)}}{\left(U_{0}+\epsilon_{1}\right)^{2}}\left|\frac{\mathscr{B}_{\mu 0}}{\epsilon_{\mu}} \sum_{n} \frac{t_{0 n} t_{n 1}}{\epsilon_{n}}\right|^{2} \\
& -\frac{16 U_{0 \mu 1 \mu}^{(2)}}{\left(U_{0}+\epsilon_{1}\right)\left(U_{0}+\epsilon_{\mu}\right)} \frac{\mathscr{L}_{\mu 0}}{\epsilon_{\mu}} \sum_{n} \frac{t_{0 n} t_{n 1}}{\epsilon_{n}} \\
& \times\left[\frac{\mathscr{L}_{0 \mu}}{\epsilon_{\mu}} \sum_{n} \frac{t_{0 n}^{2}-t_{\mu n}^{2}}{\epsilon_{n}}-\frac{\mathscr{B}_{1 \mu}}{U_{0}+\epsilon_{1}} \sum_{n} \frac{t_{0 n} t_{n 1}}{\epsilon_{n}}\right]
\end{aligned}
$$

It remains to insert here the explicit expressions for the hopping terms, Eqs. (34), in conjunction with the expressions for 
TABLE II. Values of $(\alpha \delta \mid \beta \gamma)=U_{\alpha \beta \gamma \delta}$ in terms of the Racah coefficients, taken from Table A26 of Ref. 20 (Ref. 21).

\begin{tabular}{cccc}
\hline \hline & $\alpha=x$ & $\alpha=y$ & $\alpha=z$ \\
$(\alpha 0 \mid \alpha 0)=$ & $3 B+C$ & $3 B+C$ & $C$ \\
$(\alpha 0 \mid \alpha 1)=$ & $-\sqrt{3} B$ & $\sqrt{3} B$ & 0 \\
$(\alpha 1 \mid \alpha 1)=$ & $B+C$ & $B+C$ & $4 B+C$ \\
\hline \hline
\end{tabular}

the angular momentum matrix elements, $\mathscr{L}_{\alpha \beta}$, Eqs. (8). To be specific, we consider a bond along $x$, and use the values of $U_{\alpha \beta \gamma \delta} \equiv(\alpha \delta \mid \beta \gamma)$ as listed in Table II. One then finds

$$
\begin{aligned}
& J_{x x}=-\frac{2 \lambda^{2}(3 B+C)}{\left(U_{0}+\epsilon_{x}\right)^{2}}\left[-\frac{\sqrt{3}}{U_{0}+\epsilon_{1}} \frac{t_{0 p_{x}} t_{1 p_{x}}}{\epsilon_{p_{x}}}+\frac{1}{\epsilon_{x}} \frac{t_{0 p_{x}}^{2}}{\epsilon_{p_{x}}}\right]^{2} \\
& -\frac{2 \lambda^{2}(B+C)}{\left(U_{0}+\epsilon_{1}\right)^{2}}\left[\frac{1}{\epsilon_{x}} \frac{t_{0 p_{x}} t_{1 p_{x}}}{\epsilon_{p_{x}}}\right]^{2} \\
& -\frac{4 \lambda^{2}(-B \sqrt{3})}{\left(U_{0}+\epsilon_{1}\right)\left(U_{0}+\epsilon_{x}\right)} \frac{1}{\epsilon_{x}} \frac{t_{0 p_{x}} t_{1 p_{x}}}{\epsilon_{p_{x}}} \\
& \times\left[\frac{1}{\epsilon_{x}} \frac{t_{0 p_{x}}^{2}}{\epsilon_{p_{x}}}-\frac{\sqrt{3}}{U_{0}+\epsilon_{1}} \frac{t_{0 p_{x}} t_{1 p_{x}}}{\epsilon_{p_{x}}}\right] \text {; } \\
& J_{y y}=-\frac{2 \lambda^{2}(3 B+C)}{\left(U_{0}+\epsilon_{y}\right)^{2}}\left[\frac{\sqrt{3}}{U_{0}+\epsilon_{1}} \frac{t_{0 p_{x}} t_{1 p_{x}}}{\epsilon_{p_{x}}}+\frac{1}{\boldsymbol{\epsilon}_{y}}\left(\frac{t_{0 p_{x}}^{2}}{\epsilon_{p_{x}}}-\frac{t_{y p_{z}}^{2}}{\boldsymbol{\epsilon}_{p_{z}}}\right)\right]^{2} \\
& -\frac{2 \lambda^{2}(B+C)}{\left(U_{0}+\epsilon_{1}\right)^{2}}\left[\frac{1}{\epsilon_{y}} \frac{t_{0 p_{x}} t_{1 p_{x}}}{\epsilon_{p_{x}}}\right]^{2} \\
& -\frac{4 \lambda^{2}(B \sqrt{3})}{\left(U_{0}+\epsilon_{1}\right)\left(U_{0}+\epsilon_{y}\right)} \frac{1}{\epsilon_{y}} \frac{t_{0 p_{x}} t_{1 p_{x}}}{\epsilon_{p_{x}}}\left[\frac{1}{\epsilon_{y}}\left(\frac{t_{0 p_{x}}^{2}}{\epsilon_{p_{x}}}-\frac{t_{y p_{z}}^{2}}{\epsilon_{p_{z}}}\right)\right. \\
& \left.+\frac{\sqrt{3}}{U_{0}+\epsilon_{1}} \frac{t_{0 p_{x}} t_{1 p_{x}}}{\epsilon_{p_{x}}}\right] \\
& J_{z z}=-\frac{8 \lambda^{2} C}{\left(U_{0}+\epsilon_{z}\right)^{2}}\left[\frac{1}{\epsilon_{z}}\left(\frac{t_{0 p_{x}}^{2}}{\epsilon_{p_{x}}}-\frac{t_{z p_{y}}^{2}}{\epsilon_{p_{y}}}\right)\right]^{2} \\
& -\frac{8 \lambda^{2}(4 B+C)}{\left(U_{0}+\epsilon_{1}\right)^{2}}\left[\frac{1}{\epsilon_{z}} \frac{t_{0 p_{x}} t_{1 p_{x}}}{\epsilon_{p_{x}}}\right]^{2} .
\end{aligned}
$$

An alternative derivation of these results is given in the Appendix.

One should keep in mind that Eq. (25) holds for a single bond. To obtain the effective magnetic Hamiltonian of the entire $\mathrm{CuO}_{2}$ plane, one has to sum the magnetic interaction $\mathscr{H}(i, j)$ over all bonds, allowing for the crystal symmetry. Within a classical approximation the resulting exchange Hamiltonian of the crystal has only an easy-plane anisotropy. To obtain the fourfold anisotropy within the easy plane requires a consideration of the spin-wave zero-point motion. ${ }^{7}$

Now we evaluate these results numerically. In this connection it is useful to emphasize that for their less general model YHAE have shown that the perturbative results for the anisotropy in the $J$ 's agrees to within about $10 \%$ with the numerical evaluations of exact diagonalization within a $\mathrm{Cu}$ $\mathrm{O}-\mathrm{Cu}$ cluster. For our numerical evaluation we use the parameters of Table I. We note that all the nonzero hopping matrix elements can be expressed in terms of $(p d \sigma)$ and $(p d \pi) \approx-\frac{1}{2}(p d \sigma):^{26}$

$$
t_{0, p_{x}}=-\sqrt{3} t_{1, p_{x}}=\sqrt{\frac{3}{2}}(p d \sigma), \quad t_{y, p_{z}}=t_{0, p_{y}}=(p d \pi) .
$$

Thereby we find (in $\mu \mathrm{eV}$ )

$$
\Delta J \equiv J_{\mathrm{av}}-J_{\mathrm{zz}}=30, \quad \delta J \equiv J_{\perp}-J_{\|}=41,
$$

where $J_{\mathrm{av}}=\left(J_{x x}+J_{y y}\right) / 2, J_{\perp}=J_{y y}$, and $J_{\|}=J_{x x}$. Note that the gap in the spin-wave spectrum due to the easy-plane anisotropy is proportional to $(\Delta J)^{1 / 2}$ whereas, as explained by YHAE, the gap due to the anisotropy within the basal plane is proportional to $\delta J$. (This statement applies to systems like $\mathrm{YB}_{2} \mathrm{Cu}_{3} \mathrm{O}_{6}$. In $\mathrm{Sr}_{2} \mathrm{CuO}_{2} \mathrm{Cl}_{2}$ the in-plane anisotropy will have contributions from dipolar interactions.) In YHAE, the terms in $J_{x x}$ and $J_{y y}$ involving $B \sqrt{3}$ were not included because only Coulomb matrix elements involving at most two orbitals were kept. To see the effect of the additional terms in the present work, we give, for comparison, the perturbative results of YHAE: $\Delta J=30 \mu \mathrm{eV}$ and $\delta J=26 \mu \mathrm{eV}$. (The results from exact diagonalization on a $\mathrm{Cu}-\mathrm{O}-\mathrm{Cu}$ cluster were $\Delta J=31 \mu \mathrm{eV}$ and $\delta J=31 \mu \mathrm{eV}$.)

\section{CONCLUSIONS}

In view of the results of Ref. 4, YHAE already demonstrated that the out-of-plane anisotropy in the superexchange interaction between $\mathrm{Cu}$ ions is dominated by Coulomb exchange terms. Therefore, the simplified Coulomb interaction of Eq. (9), used widely in the literature, is insufficient to explain this anisotropy. YHAE then considered the anisotropy due to the simplest exchange terms, like $U_{\alpha \beta \beta \alpha}$, which involve only two orbitals, and thereby obtained an anisotropy in the superexchange interaction whose value agreed with experiments.

In the present paper we included all the Coulomb terms which are allowed by tetragonal site symmetry, and found that the additional terms, involving ( $\alpha 0 \mid \alpha 1)$, practically do not affect the out-of-plane anisotropy $\Delta J$. The in-plane single-bond anisotropy, $\delta J$, is somewhat larger than before. YHAE showed that the in-plane gap in the spin-wave spectrum is a manifestation of quantum zero-point fluctuations and is proportional to $\delta J$. Using the results of our present calculation in Eq. (82) of YHAE we estimate the in-plane gap in the spin-wave spectrum to be about $33 \mu \mathrm{eV} \sim 0.27$ $\mathrm{cm}^{-1}$. The direct observation of this gap would provide an interesting and significant test of our calculations.

\section{ACKNOWLEDGMENTS}

We acknowledge support from the U.S.-Israel Binational Science Foundation. A.B.H. was also supported in part by the U.S. Israel Education Foundation and by the National Science Foundation under Grant No. DMR-91-22784. 


\section{APPENDIX: ALTERNATIVE CALCULATION OF THE EXCHANGE ANISOTROPY}

In this appendix we obtain the result for $J_{\mu \mu}$ for tetragonal symmetry by direct application of perturbation theory. We take the unperturbed Hamiltonian to be the same as that of Eq. (9), except that we do not include in it the spin-orbit interaction on the copper ions. [See Eq. (2).] That term is now to be included into $\mathscr{H}_{1}$. For simplicity we only consider the contribution to the anisotropy from channel $a$. A similar calculation to that given here shows that channel $b$ gives no anisotropy. Since we only consider channel $a$, we can eliminate the oxygens completely from the problem by introducing an effective hopping matrix element $t_{\alpha \beta}$ between crystal-field states on near-neighboring copper ions. For instance,

$$
\begin{gathered}
\bar{t}_{00}=t_{0, p_{x}}^{2} / \epsilon_{p_{x}}, \quad \bar{t}_{01}=t_{0, p_{x}} t_{1, p_{x}} / \epsilon_{p_{x}}, \quad \bar{t}_{x x}=0, \\
\bar{t}_{y y}=t_{y, p_{z}}^{2} / \epsilon_{p_{z}}, \quad \bar{t}_{z z}=t_{z, p_{y}}^{2} / \epsilon_{p_{y}},
\end{gathered}
$$

as in Eq. (53) of YHAE. Accordingly, we need to work to fifth order in perturbation theory, where the perturbations are $V$, the spin-orbit interaction, which we take to second order, $\mathscr{H}_{\text {hop }} \equiv \mathscr{T}$, the hopping between copper ions, which we take to second order, and $\Delta \mathscr{H}_{C}=\mathscr{C}$ on the copper ions, which we take to first order. We also use the fact that $U_{\alpha \beta \gamma \delta}$ is only nonzero when $\sigma(\alpha) \sigma(\beta) \sim \sigma(\gamma) \sigma(\delta)$.

In fifth-order perturbation theory there are, a priori, 30 different ways to order these perturbations. But obviously the Coulomb perturbation can only appear when the two holes are on the same site. So we have only to consider how to insert two powers of $V$ into the sequence $\mathscr{T} \mathscr{C} \mathscr{T}$. There are basically three types of terms to consider. The first is

$$
H_{1} \equiv\left(V \frac{1}{\mathscr{E}} \mathscr{T}+\mathscr{T} \frac{1}{\mathscr{E}} V\right) \frac{1}{\mathscr{E}} \mathscr{C} \frac{1}{\mathscr{E}}\left(V \frac{1}{\mathscr{E}} \mathscr{T}+\mathscr{T} \frac{1}{\mathscr{E}} V\right),
$$

where $\mathscr{E}$ denotes the appropriate energy denominator. The second type of terms are those in which the two $V$ 's are both to the left of $\mathscr{C}$, and the third type are terms which are the Hermitian conjugates of the second type, i. e., those in which the two $V$ 's are to the right of $\mathscr{C}$.

That the second type of term does not lead to any anisotropy can be established by the following argument. Suppose we show that these terms vanish when applied to any triplet spin state. That would imply that these terms are of the form $(1 / 4)-\mathbf{S}_{i} \cdot \mathbf{S}_{j}$, which obviously gives rise to no anisotropy. The second type of term has first (reading from right to left) $\mathscr{T}$ acting on the triplet state. That will put the two holes, which were initially distributed one on site $i$, the other on site $j$, onto the same site, perforce one in state $|0\rangle$, the other in state $|1\rangle$. Now apply the Coulomb perturbation. This operator can leave the two holes in the same states, viz. one in $|0\rangle$ and the other in $|1\rangle$. In fact, by our observation on the form of $U_{\alpha \beta \gamma \delta}$ and by the fact that parallel spins are not allowed in the same spatial orbital, there are no other final states. But such a diagonal matrix element of $\mathbf{U}$ was treated by YHAE and found to give no anisotropy in the second type of term. So we conclude that the second type of term does not give rise to any anisotropy. Terms of the third type are the Hermitian conjugate of type two and therefore are subject to the same argument. Thus all the anisotropic terms are contained in the expression in Eq. (A2).

If $T_{i j}$ denotes hopping from $i$ to $j$, we can write Eq. (A2) as

$$
H_{1}=2 \sum_{\alpha=x, y, z} \mathbf{Q}_{\alpha}^{\dagger} \frac{1}{\mathscr{E}} C \frac{1}{\mathscr{E}} \mathbf{Q}_{\alpha},
$$

where

$$
\mathbf{Q}_{\alpha}=\left(V_{\alpha} \frac{1}{\mathscr{E}} T_{i j}+T_{i j} \frac{1}{\mathscr{E}} V_{\alpha}\right) .
$$

Here $V_{\alpha}=\Sigma_{h} L_{\alpha}(h) s_{\alpha}(h)$, where the sum is over the two holes, $h$. Acting on the ground state, the operator $\mathbf{Q}_{\alpha}$ produces two final states, depending on whether the orbital $|0\rangle$ or $|1\rangle$ is occupied. So we define

$$
\begin{aligned}
{\left[Q_{\alpha}^{(\gamma)}\right]_{\sigma, \eta ; \sigma^{\prime}, \eta^{\prime}}=} & \langle 0| d_{i, \gamma, \sigma} d_{i, \alpha, \eta}\left(V_{\alpha} \frac{1}{\mathscr{E}} T_{i j}\right. \\
& \left.+T_{i j} \frac{1}{\mathscr{E}} V_{\alpha}\right) d_{i, 0, \eta^{\prime}}^{\dagger} d_{j, 0, \sigma^{\prime}}^{\dagger}|0\rangle,
\end{aligned}
$$

where $\gamma$ can be 0 or 1 . Then

$$
H_{1}=2 \sum_{\alpha \gamma \gamma^{\prime}}\left[\mathbf{Q}_{\alpha}^{\left(\gamma^{\prime}\right)}\right]^{\dagger}\left[\mathscr{C}_{\alpha}^{\gamma^{\prime} \gamma}\right]\left[\mathbf{Q}_{\alpha}^{(\gamma)}\right] \Delta_{\gamma \alpha}^{-1} \Delta_{\gamma^{\prime} \alpha}^{-1},
$$

where $\Delta_{\gamma \alpha}=\epsilon_{\gamma}+\epsilon_{\alpha}+U_{0}$ and

$$
\begin{aligned}
{\left[\mathscr{C}_{\alpha}^{\gamma^{\prime} \gamma}\right]_{\sigma^{\prime \prime} \eta^{\prime \prime} ; \sigma^{\prime} \eta^{\prime}}=} & \left\langle 0\left|d_{i \gamma^{\prime} \sigma^{\prime \prime}} d_{i \alpha \eta^{\prime \prime}} \Delta \mathscr{H}_{C} d_{i \alpha \eta^{\prime}}^{\dagger} d_{i \gamma \sigma^{\prime}}^{\dagger}\right| 0\right\rangle \\
= & \delta_{\eta^{\prime \prime}, \eta^{\prime}} \delta_{\sigma^{\prime}, \sigma^{\prime \prime}}\left\langle\alpha \gamma^{\prime}\left|\Delta \mathscr{H}_{C}\right| \alpha \gamma\right\rangle \\
& -\delta_{\eta^{\prime \prime}, \sigma^{\prime}} \delta_{\sigma^{\prime \prime}, \eta^{\prime}}\left\langle\alpha \gamma^{\prime}\left|\Delta \mathscr{H}_{C}\right| \gamma \alpha\right\rangle .
\end{aligned}
$$

Now we introduce the notation for direct products

$$
[\mathscr{C} \mathscr{B}]_{\sigma^{\prime}, \eta^{\prime} ; \sigma, \eta}=A_{\sigma^{\prime} \sigma} B_{\eta^{\prime} \eta},
$$

so that

$$
\mathscr{C}_{\alpha}^{\gamma^{\prime}, \gamma}=[\odot]\left\langle\alpha \gamma^{\prime}\left|\Delta \mathscr{H}_{C}\right| \alpha \gamma\right\rangle \equiv[\odot]\left(\alpha \gamma \mid \alpha \gamma^{\prime}\right)
$$

in the notation of Eqs. (3). Also $[\mathscr{G}=[\mathscr{T} \mathscr{T}-\vec{\sigma} \cdot \vec{\sigma}] / 2$.

From Appendix $\mathrm{H}$ of YHAE we also take the results

$$
\left[\mathbf{Q}_{\alpha}^{(0)}\right]=\left(C_{1}+C_{2}\right)\left[\mathscr{T} \sigma_{\alpha}\right]-C_{2}\left[\mathscr{T} \sigma_{\alpha}\right][\odot]
$$

and

$$
\left[\mathbf{Q}_{\alpha}^{(1)}\right]=C_{3}\left[\mathscr{T} \sigma_{\alpha}\right]
$$

where

$$
C_{1}+C_{2}=\left[\frac{\left(\bar{t}_{\alpha \alpha}-\bar{t}_{00}\right) \mathscr{C}_{0 \alpha}}{\epsilon_{\alpha}}+\frac{\bar{t}_{01} \mathscr{b}_{1 \alpha}}{\left(\epsilon_{1}+U_{0}\right)}\right]
$$

and

$$
C_{3}=-\bar{t}_{01} \mathscr{L}_{0 \alpha}\left(\frac{\epsilon_{1}+\epsilon_{\alpha}+U_{0}}{\epsilon_{\alpha}\left(\epsilon_{1}+U_{0}\right)}\right) .
$$

Thus we obtain the result 


$$
\begin{aligned}
\mathscr{H}(i, j)= & \sum_{\alpha}\left\{\frac{2(\alpha 0 \mid \alpha 0)}{\Delta_{0}^{2}}\left[C_{1}^{*} \mathscr{T} \mathscr{T}+C_{2}^{*} \mathscr{T} \mathscr{T}-C_{2}^{*} \mathscr{O}\right]\left[\mathscr{T} \sigma_{\alpha}\right][\mathscr{O}]\left[\mathscr{T} \sigma_{\alpha}\right]\left[C_{1} \mathscr{T} \mathscr{T}+C_{2} \mathscr{T} \mathscr{T}-C_{2} \mathscr{O}\right]\right. \\
& +\frac{2(\alpha 1 \mid \alpha 1)}{\Delta_{1}^{2}}\left(C_{3}^{*}\left[\mathscr{T} \sigma_{\alpha}\right][\mathscr{O}]\left[\mathscr{T} \sigma_{\alpha}\right] C_{3}\right)+\frac{2(\alpha 0 \mid \alpha 1)}{\Delta_{0} \Delta_{1}} C_{3}^{*}\left[\mathscr{T} \sigma_{\alpha}\right][\mathscr{O}]\left[\mathscr{T} \sigma_{\alpha}\right]\left[C_{1} \mathscr{T} \mathscr{T}+C_{2} \mathscr{T} \mathscr{T}-C_{2} \mathscr{O}\right] \\
& \left.+\frac{2(\alpha 0 \mid \alpha 1)}{\Delta_{0} \Delta_{1}}\left[C_{1}^{*} \mathscr{T} \mathscr{T}+C_{2}^{*} \mathscr{T} \mathscr{T}-C_{2}^{*} \mathscr{O}\right]\left[\mathscr{T} \sigma_{\alpha}\right][\mathscr{O}]\left[\mathscr{T} \sigma_{\alpha}\right] C_{3}\right\} .
\end{aligned}
$$

Now we use the equality

$$
\left[\mathscr{T} \sigma_{\alpha}\right][\mathscr{O}]\left[\mathscr{T} \sigma_{\alpha}\right]=\frac{1}{2}\left[\mathscr{T} \mathscr{T}-2 \sigma_{\alpha} \sigma_{\alpha}+\vec{\sigma} \cdot \vec{\sigma}\right]
$$

So, dropping isotropic terms, we have $\mathrm{e}^{21}$

$$
\begin{aligned}
\mathscr{H}(i, j)= & \sum_{\alpha}\left\{-\frac{2(\alpha 0 \mid \alpha 0)}{\Delta_{0 \alpha}^{2}}\left[C_{1}^{*} \mathscr{T} \mathscr{T}+C_{2}^{*} \mathscr{T} \mathscr{T}-C_{2}^{*} \mathscr{O}\right]\left[\sigma_{\alpha} \sigma_{\alpha}\right]\left[C_{1} \mathscr{T} \mathscr{T}+C_{2} \mathscr{T} \mathscr{T}-C_{2} \mathscr{O}\right]-\frac{2(\alpha 1 \mid \alpha 1)}{\Delta_{1 \alpha}^{2}} C_{3}^{*}\left[\sigma_{\alpha} \sigma_{\alpha}\right] C_{3}\right. \\
& \left.-\frac{2(\alpha 0 \mid \alpha 1)}{\Delta_{0 \alpha} \Delta_{1 \alpha}} C_{3}^{*}\left[\sigma_{\alpha} \sigma_{\alpha}\right]\left[C_{1} \mathscr{T} \mathscr{T}+C_{2} \mathscr{T} \mathscr{T}-C_{2} \mathscr{O}\right]-\frac{2(\alpha 1 \mid \alpha 0)}{\Delta_{0 \alpha} \Delta_{1 \alpha}}\left[C_{1}^{*} \mathscr{T} \mathscr{T}+C_{2}^{*} \mathscr{T} \mathscr{T}-C_{2}^{*} \mathscr{O}\right]\left[\sigma_{\alpha} \sigma_{\alpha}\right] C_{3}\right\} .
\end{aligned}
$$

Now use

$$
2\left[\sigma_{\alpha} \sigma_{\alpha}\right][\mathscr{O}]=2[\mathscr{O}]\left[\sigma_{\alpha} \sigma_{\alpha}\right]=[\vec{\sigma} \cdot \vec{\sigma}-\mathscr{T} \mathscr{T}] .
$$

Thus the terms involving $\mathscr{O}$ are isotropic. So the anisotropic terms are correctly given by Eq. (26) with

$$
J_{\mu \mu}=-\frac{8(\mu 0 \mid \mu 0)}{\Delta_{0 \mu}^{2}}\left|C_{1}+C_{2}\right|^{2}-\frac{8(\mu 1 \mid \mu 1)}{\Delta_{1 \mu}^{2}}\left|C_{3}\right|^{2}-16 \operatorname{Re} \frac{(\mu 0 \mid \mu 1)}{\Delta_{0 \mu} \Delta_{1 \mu}} C_{3}^{*}\left(C_{1}+C_{2}\right) .
$$

Now we use Eqs. (A12) and (A13) to write

$$
\begin{aligned}
J_{\mu \mu}= & \frac{8(\mu 0 \mid \mu 0)}{\left(U_{0}+\epsilon_{\mu}\right)^{2}}\left[\frac{\left(\bar{t}_{00}-\bar{t}_{\mu \mu}\right) \mathscr{L}_{0 \mu}}{\epsilon_{\mu}}-\frac{\bar{t}_{01} \mathscr{L}_{1 \mu}}{\left(\epsilon_{1}+U_{0}\right)}\right]^{2}+\frac{8(\mu 1 \mid \mu 1)}{\epsilon_{\mu}^{2}\left(\epsilon_{1}+U_{0}\right)^{2}} \vec{t}_{01}^{2} \mathscr{L}_{0 \mu}^{2}-\frac{16(\mu 0 \mid \mu 1) \bar{t}_{10} \mathscr{L}_{0 \mu}}{\epsilon_{\mu}\left(U_{0}+\epsilon_{\mu}\right)\left(\epsilon_{1}+U_{0}\right)} \\
& \times\left[\frac{\left(\bar{t}_{\mu \mu}-\bar{t}_{00}\right) \mathscr{L}_{0 \mu}}{\epsilon_{\mu}}+\frac{\bar{t}_{01} \mathscr{L}_{1 \mu}}{\left(\epsilon_{1}+U_{0}\right)}\right] .
\end{aligned}
$$

This result reproduces Eqs. (40) of the text.

${ }^{1}$ D. Coffey, K. S. Bedell, and S. A. Trugman, Phys. Rev. B 42, 6509 (1990).

${ }^{2}$ N. E. Bonesteel, Phys. Rev. B 47, 9144 (1993).

${ }^{3}$ L. Shekhtman, A. Aharony, and O. Entin-Wohlman, Phys. Rev. B 47, 174 (1993); O. Entin-Wohlman, A. Aharony, and L. Shekhtman, ibid. 50, 3068 (1994).

${ }^{4}$ F. Barriquand and G. A. Sawatzky, Phys. Rev. B 50, 16649 (1994).

${ }^{5}$ W. Koshibae, Y. Ohta, and S. Maekawa, Phys. Rev. Lett. 71, 467 (1993); Phys. Rev. B 50, 3767 (1994).

${ }^{6}$ T. Yildirim, A. B. Harris, O. Entin-Wohlman, and A. Aharony, Phys. Rev. Lett. 73, 2919 (1994).

${ }^{7}$ T. Yildirim, A. B. Harris, A. Aharony, and O. Entin-Wohlman, Phys. Rev. B 52, 10239 (1995).

${ }^{8}$ For additional references, see Ref. 7.

${ }^{9}$ R. J. Birgeneau and G. Shirane, in Physical Properties of High Temperature Superconductors I, edited by D. M. Ginsberg (World Scientific, Singapore, 1989), and references therein.
${ }^{10}$ C. J. Peters, R. J. Birgeneau, M. A. Kastner, H. Yoshizawa, Y. Endoh, J. Tranquada, G. Shirane, Y. Hidaka, M. Oda, M. Suzuki, and T. Murakami, Phys. Rev. B 37, 9761 (1988).

${ }^{11}$ T. Thio, T. R. Thurston, N. W. Preyer, P. J. Picone, M. A. Kastner, H. P. Henssen, D. R. Gabbe, C. Y. Chen, R. J. Birgeneau, and A. Aharony, Phys. Rev. B 38, 905 (1988).

${ }^{12}$ D. Vaknin, S. K. Sinha, C. Stassis, L. L. Miller, and D. C. Johnston, Phys. Rev. B 41, 1926 (1990).

${ }^{13}$ M. Greven, R. J. Birgeneau, Y. Endoh, M. A. Kastner, M. Matsuda, and G. Shirane, Phys. Rev. Lett. 74, 1096 (1994).

${ }^{14}$ P. Bourges, L. Boudarene, D. Petitgrand, and P. Galez, Physica B 180 \& 181, 447 (1992); P. Bourges, A. S. Ivanov, D. Petitgrand, J. Rossat-Mignod, and L. Boudarene, ibid. 186-188, 925 (1993).

${ }^{15}$ I. W. Sumarlin, J. W. Lynn, T. Chattopadhyay, S. N. Barilo, D. I. Zhigunov, and J. L. Peng, Phys. Rev. B 51, 5824 (1995).

${ }^{16}$ B. Keimer, R. J. Birgeneau, A. Cassanho, Y. Endoh, M. Greven, M. A. Kastner, and G. Shirane, Z. Phys. B 91, 373 (1993). 
${ }^{17}$ P. W. Anderson, Phys. Rev. 115, 2 (1959).

${ }^{18}$ T. Moriya, Phys. Rev. 120, 91 (1960).

${ }^{19}$ I. Dzyaloshinskii, J. Phys. Chem. Solids 4, 241 (1958).

${ }^{20}$ J. S. Griffith, The Theory of Transition Metal Ions (Cambridge University Press, Cambridge, 1961).

${ }^{21}$ The states $\epsilon, \theta, \xi, \eta$, and $\zeta$ in Table A26 of Ref. 20 should be identified with our states $|0\rangle,|1\rangle,|x\rangle,|y\rangle$, and $|z\rangle$, respectively.

${ }^{22}$ J. Stein, O. Entin-Wohlman, and A. Aharony, Phys. Rev. B (to be published).

${ }^{23}$ H. Eskes and J. H. Jefferson, Phys. Rev. B 48, 9788 (1993).

${ }^{24}$ M. Takahashi, J. Phys. C 10, 1289 (1971).

${ }^{25}$ J. B. Grant and A. K. McMahan, Phys. Rev. B 46, 8440 (1992).

${ }^{26}$ L. F. Matheiss, Phys. Rev. B 5, 290 (1972). 